Revista Destaques Acadêmicos, Lajeado, v. 13, n. 2, 2021. ISSN 2176-3070

DOI: http://dx.doi.org/10.22410/issn.2176-3070.v13i2a2021.2596

http://www.univates.br/revistas

\title{
IDENTIFICAÇÃO DE TALENTOS ESPORTIVOS NA ESCOLA
}

\author{
Igor Joel da Costa Francisco ${ }^{1}$, Rodrigo Lara Rother ${ }^{2}$
}

Resumo: A descoberta de talentos no esporte é um terreno fértil para investigações, ainda mais no Brasil, onde se busca á muitos anos encontrar uma forma sistemática para descobrir e desenvolver estes talentos. Ainda assim, o Programa Esporte Brasil (PROESP-BR) oferece uma bateria de testes e valores referência para serem usados de forma voluntária no país. O presente estudo buscou identificar possíveis talentos para o esporte na escola, avaliando-se a massa corporal, estatura, IMC e cinco testes de aptidão física em 73 escolares, meninos e meninas, de 12 a 14 anos. Entre os avaliados, somente $2,19 \%$ apresentaram resultados considerados "excelentes" em pelo menos um dos testes, estando concentrados nos testes de força explosiva de membros superiores e no teste de aptidão cardiorrespiratória. Conclui-se que ações voltadas ao treinamento esportivo devam ser voltadas a modalidades caracterizadas pelas duas habilidades identificadas e, para os demais, sugerem-se ações voltadas para a saúde, já que a grande maioria apresentou resultados abaixo dos valores indicados para se ter uma vida saudável.

Palavras-chave: Educação Física Escolar, Aptidão Física, Esporte.

\section{INTRODUÇÃO}

Trata-se por Talento Esportivo aquele indivíduo que possui características ou habilidades herdadas ou adquiridas, uma aptidão especial acima da média para o desenvolvimento esportivo de alguma modalidade (BÖHME, 1999). Para se formar um Talento Esportivo existem métodos e procedimentos, uma dessas formas é o Treinamento de Longo Prazo (TLP). Esse processo se divide em quatro etapas, sendo elas a detecção, formação, seleção e promoção do talento.

A detecção é a primeira parte, correspondendo a todas as formas que são utilizadas para encontrar crianças e adolescentes dispostos a ingressar em um programa de formação esportiva básica (BENDA, 1998). Segundo Queiroz e Fernández (2010) o método de detecção deve estar ligado a um programa

1 Graduado, Educação Física - Licenciatura, UNIVATES, igorfrancisco@universo.univates.br

2 Doutor em Ciências: Ambiente e Desenvolvimento, Docente do curso de Educação Física, UNIVATES, rodrigorother@univates.br 
de desenvolvimento e ter uma sustentação científica, direcionado por índices e metas que devem ser atingidas.

A segunda parte é a formação, onde se busca desenvolver as qualidades do talento com profissionais e treinamento, após há a etapa de seleção, que são os meios utilizados para escolher quais destes talentos possuem condições mais altas de treinamento e desempenho. Por último, vem a etapa de promoção que se trata da utilização de procedimentos de treinamentos e qualquer outro recurso necessário para aumentar o desempenho e desenvolvimento dos talentos que foram escolhidos, promovendo-os ao alto nível do esporte (BÖHME, 1999).

Tratando mais especificamente da primeira etapa, chama a atenção que no Brasil não há uma forma sistemática e organizada para detectar o talento esportivo, sendo que cada modalidade realiza seus próprios processos da forma que acha mais adequado, o que nem sempre leva a obtenção de resultados satisfatórios (ROTHER, 2015). Apesar de no país não haver essa sistematização para descobrir os talentos, existe um programa governamental, voluntário, chamado Projeto Esporte Brasil (PROESP-BR), que tem a intenção de caminhar nesse sentido. O projeto ajuda no descobrimento de possíveis talentos através de testes físicos, critérios e normas de avaliação e valores de referência disponíveis de forma virtual. Com base nisso o professor pode avaliar seus alunos voluntariamente e identificar se há algum talento para alguma modalidade específica dentre seus alunos.

Desta forma, o presente estudo tem o objetivo de identificar possíveis talentos para o esporte e sugerir modalidades adequadas aos escolares avaliados de acordo com suas características morfológicas e motoras. Para isso, foram utilizadas avaliações sugeridas pelo PROESP-BR, traçando o perfil antropométrico e avaliando o desempenho de escolares meninos e meninas, com idade entre 12 e 14 anos.

\section{MÉTODOS}

A presente pesquisa pode ser classificada como quantitativa de caráter descritivo. Todos os dados foram coletados com 73 alunos de uma mesma escola municipal de ensino fundamental, de um município do interior do Rio Grande do Sul. A amostra foi constituída por meninos (39) e meninas (34) com idade entre 12 e 14 anos.

Foram considerados como critérios de inclusão da amostra ter idade entre 12 e 14 anos, não possuir qualquer tipo de deficiência física que impossibilitasse a aplicação dos testes e apresentar o Termo de Consentimento Livre e Esclarecido assinado pelos pais e/ou responsáveis.

Foram feitas cinco visitas a escola. Na primeira visita foi realizada a pesagem das crianças sobre uma balança portátil (marca Clinck e modelo QF - 2003 D) sendo anotado o seu peso em quilogramas e medida a estatura, 
utilizando de uma fita métrica (marca Snauzer) afixada em parede plana. De posse desses dados, calculou-se o Índice de Massa Corporal - IMC.

Nas outras idas a escola, foram realizados na quadra do ginásio esportivo os testes de Força Explosiva de Membros Inferiores (salto horizontal), de Velocidade de Deslocamento (corrida de 20 metros), de Agilidade (teste do quadrado), de Força Explosiva de Membros Superiores (arremesso do medicinebal) e de Aptidão Cardiorrespiratória (corrida/caminhada dos 6minutos), todos protocolados pelo PROESP-BR, versão 2016.

Todos os resultados foram tabulados em planilha Excel e submetidos a análise descritiva, calculando valores mínimos, máximos, médias e desvio padrão.

\section{RESULTADOS}

Os resultados obtidos nos testes foram avaliados de acordo com as tabelas de avaliação do PROESP-BR, as quais classificam os resultados como "excelente", "muito bom", "bom", "razoável" e "fraco". O IMC se divide em "baixo peso", "normal", "sobrepeso" e "obesidade".

As tabelas 1 e 2 apresentam os valores gerais de massa corporal, estatura, IMC, força explosiva de membros inferiores, velocidade de deslocamento, agilidade, força explosiva de membros superiores, aptidão cardiorrespiratória, dos avaliados com idade de 12 anos do sexo masculino e feminino.

Tabela 1 - Valores descritivos gerais das variáveis avaliadas (Meninos com 12 anos)

\begin{tabular}{l|c|c|c|c|c}
\hline \multicolumn{1}{c|}{ Variáveis } & Mínimo & Máximo & Média & Resultado & DesPad \\
\hline Peso (Kg) & 39 & 91 & 57,84 & - & 19,63 \\
\hline Estatura (m) & 1,41 & 1,68 & 1,43 & - & 0,08 \\
\hline IMC (kg/m2) & 16,23 & 33,02 & 21,88 & Sobrepeso & 5,80 \\
\hline Força Explosiva MI (cm) & 1,04 & 1,60 & 1,23 & Fraco & 0,17 \\
\hline Velocidade Desloc. (s) & 3,93 & 5,05 & 3,86 & Fraco & 0,35 \\
\hline Agilidade (s) & 6,59 & 7,43 & 6,22 & Fraco & 0,31 \\
\hline Força Explosiva MS (cm) & 2,90 & 4,49 & 2,99 & Bom & 0,56 \\
\hline Aptidão Cardiorresp. (m) & 702 & 1192 & 816,83 & Fraco & 170,54 \\
\hline
\end{tabular}

Fonte: os autores (2021).

Dos nove alunos desta idade e sexo avaliados nos cinco testes, um menino apresentou resultado "excelente" de acordo com os valores de referência do PROESP-BR. O aluno atingiu a marca de $4,49 \mathrm{~cm}$ no teste de força explosiva de membros superiores (arremesso de medicinebal). 
Tabela 2 - Valores descritivos gerais das variáveis avaliadas (Meninas com 12 anos)

\begin{tabular}{l|c|c|c|c|c}
\hline \multicolumn{1}{c|}{ Variáveis } & Mínimo & Máximo & Média & Resultado & DesPad \\
\hline Peso (Kg) & 33 & 65 & 47,61 & - & 9,55 \\
\hline Estatura (m) & 1,43 & 1,73 & 1,48 & - & 0,08 \\
\hline IMC (kg/m2) & 14,47 & 25,32 & 18,46 & Normal & 2,92 \\
\hline Força Explosiva MI (cm) & 1,02 & 1,74 & 1,26 & Razoável & 0,19 \\
\hline Velocidade Desloc. (s) & 3,90 & 5,17 & 4,09 & Fraco & 0,32 \\
\hline Agilidade (s) & 6,32 & 7,53 & 6,47 & Razoável & 0,42 \\
\hline Força Explosiva MS (cm) & 2,09 & 3,70 & 2,70 & Bom & 0,48 \\
\hline Aptidão Cardiorresp. (m) & 580 & 1062 & 757,31 & Fraco & 154,03 \\
\hline
\end{tabular}

Fonte: os autores (2021).

Foram avaliadas treze alunas desta idade e sexo, sendo que dos cinco testes, uma menina apresentou resultado "excelente" de acordo com os valores de referência do PROESP. Esta aluna atingiu a marca de $3,70 \mathrm{~cm}$ no teste de força explosiva de membros superiores (arremesso de medicinebal). Também entre as meninas chamou atenção a estatura de $1,73 \mathrm{~cm}$ que uma delas possui, pelo fato de ter apenas 12 anos de idade.

As tabelas 3 e 4 apresentam os valores gerais de massa corporal, estatura, IMC, força explosiva de membros inferiores, velocidade de deslocamento, agilidade, força explosiva de membros superiores, aptidão cardiorrespiratória, dos avaliados com idade de 13 anos do sexo masculino e feminino.

Tabela 3 - Valores descritivos gerais das variáveis avaliadas (Meninos com 13 anos)

\begin{tabular}{l|c|c|c|c|c}
\hline \multicolumn{1}{c|}{ Variáveis } & Mínimo & Máximo & Média & Resultado & DesPad \\
\hline Peso (Kg) & 35 & 68 & 51,90 & - & 10,30 \\
\hline Estatura (m) & 1,44 & 1,77 & 1,51 & - & 0,10 \\
\hline IMC (kg/m2) & 16,02 & 26,71 & 20,22 & Sobrepeso & 3,52 \\
\hline Força Explosiva MI (cm) & 1,10 & 2,14 & 1,51 & Razoável & 0,33 \\
\hline Velocidade Desloc. (s) & 3,25 & 4,76 & 3,86 & Fraco & 0,49 \\
\hline Agilidade (s) & 5,84 & 7,30 & 6,17 & Razoável & 0,49 \\
\hline Força Explosiva MS (cm) & 2,50 & 5,39 & 3,36 & Bom & 0,80 \\
\hline Aptidão Cardiorresp. (m) & 597 & 1586 & 986,98 & Razoável & 278,79 \\
\hline
\end{tabular}

Fonte: os autores (2021). 
Dos 18 alunos desta idade e sexo avaliados nos cinco testes, três meninos apresentaram resultado "excelente" de acordo com os valores de referência do PROESP. Um aluno atingiu a marca de $5,39 \mathrm{~cm}$ no teste de força explosiva de membros superiores (arremesso de medicinebal) e dois alunos atingiram a marca de $1564 \mathrm{~m}$ e $1586 \mathrm{~m}$ no teste de aptidão cardiorrespiratória (corrida/caminhada dos 6minutos). Entre estes meninos também se destacam dois alunos com estatura de 1,76 cm e 1,77 cm como os mais altos de sua idade.

Tabela 4 - Valores descritivos gerais das variáveis avaliadas (Meninas com 13 anos)

\begin{tabular}{l|c|c|c|c|c}
\hline \multicolumn{1}{c|}{ Variáveis } & Mínimo & Máximo & Média & Resultado & DesPad \\
\hline Peso $(\mathrm{Kg})$ & 31,5 & 73,5 & 49,09 & - & 11,48 \\
\hline Estatura $(\mathrm{m})$ & 1,46 & 1,66 & 1,47 & - & 0,05 \\
\hline IMC $(\mathrm{kg} / \mathrm{m} 2)$ & 14,38 & 29,07 & 19,68 & Normal & 4,13 \\
\hline Força Explosiva MI (cm) & 0,89 & 1,64 & 1,27 & Razoável & 0,20 \\
\hline Velocidade Desloc. (s) & 3,98 & 6,16 & 4,22 & Fraco & 0,57 \\
\hline Agilidade (s) & 6,06 & 7,53 & 6,45 & Razoável & 0,69 \\
\hline Força Explosiva MS (cm) & 2,20 & 4,40 & 2,95 & Bom & 0,56 \\
\hline Aptidão Cardiorresp. $(\mathrm{m})$ & 648 & 1078 & 838,85 & Razoável & 125,88 \\
\hline
\end{tabular}

Fonte: os autores (2021).

Das 15 alunas desta idade e sexo avaliadas nos cinco testes, uma menina apresentou resultado "excelente" de acordo com os valores de referência do PROESP. A aluna atingiu a marca de $4,40 \mathrm{~cm}$ no teste de força explosiva de membros superiores (arremesso de medicinebal).

As tabelas 5 e 6 apresentam os valores gerais de massa corporal, estatura, IMC, força explosiva de membros inferiores, velocidade de deslocamento, agilidade, força explosiva de membros superiores, aptidão cardiorrespiratória, dos avaliados com idade de 14 anos do sexo masculino e feminino.

Dos 12 alunos desta idade e sexo avaliados nos cinco testes, dois meninos apresentaram resultado "excelente" de acordo com os valores de referência do PROESP. Os alunos atingiram a marca de $1429 \mathrm{~m}$ e $1472 \mathrm{~m}$ no teste de aptidão cardiorrespiratória (corrida/caminhada dos 6minutos). 
Tabela 5 - Valores descritivos gerais das variáveis avaliadas (Meninos com 14 anos)

\begin{tabular}{l|c|c|c|c|c}
\hline \multicolumn{1}{c|}{ Variáveis } & Mínimo & Máximo & Média & Resultado & DesPad \\
\hline Peso $(\mathrm{Kg})$ & 38 & 76 & 51,82 & - & 10,94 \\
\hline Estatura $(\mathrm{m})$ & 1,54 & 1,77 & 1,50 & - & 0,06 \\
\hline IMC $(\mathrm{kg} / \mathrm{m} 2)$ & 15,61 & 28,08 & 19,43 & Normal & 4,07 \\
\hline Força Explosiva MI (cm) & 1,25 & 2,07 & 1,54 & Fraco & 0,21 \\
\hline Velocidade Desloc. (s) & 3,48 & 5,35 & 3,72 & Fraco & 0,54 \\
\hline Agilidade (s) & 5,71 & 6,96 & 5,91 & Fraco & 0,39 \\
\hline Força Explosiva MS (cm) & 3,11 & 4,64 & 3,44 & Razoável & 0,50 \\
\hline Aptidão Cardiorresp. (m) & 782 & 1472 & 1098,12 & Bom & 216,47 \\
\hline
\end{tabular}

Fonte: os autores (2021).

Tabela 6 - Valores descritivos gerais das variáveis avaliadas (Meninas com 14 anos)

\begin{tabular}{l|c|c|c|c|c}
\hline \multicolumn{1}{c|}{ Variáveis } & Mínimo & Máximo & Média & Resultado & DesPad \\
\hline Peso $(\mathrm{Kg})$ & 47 & 66 & 46,24 & - & 6,47 \\
\hline Estatura $(\mathrm{m})$ & 1,52 & 1,66 & 1,30 & - & 0,05 \\
\hline IMC $(\mathrm{kg} / \mathrm{m} 2)$ & 20,34 & 25,46 & 18,94 & Normal & 2,00 \\
\hline Força Explosiva MI (cm) & 1,02 & 1,33 & 0,99 & Fraco & 0,12 \\
\hline Velocidade Desloc. (s) & 4,30 & 6,09 & 4,15 & Fraco & 0,68 \\
\hline Agilidade (s) & 6,09 & 8,32 & 5,95 & Fraco & 0,92 \\
\hline Força Explosiva MS (cm) & 2,80 & 3,44 & 2,60 & Razoável & 0,25 \\
\hline Aptidão Cardiorresp. $(\mathrm{m})$ & 693 & 935 & 717,21 & Fraco & 105,29 \\
\hline
\end{tabular}

Fonte: os autores (2021).

Entre as seis alunas avaliadas desta idade e sexo, nenhuma apresentou resultado "excelente" em nenhum dos cinco testes.

No cálculo de IMC dos 73 avaliados, foram classificados como "baixo peso" (1,37\%), 41 "normais" (56,16\%), 24 "sobrepesos" (32,88\%) e sete "obesos" $(9,59 \%)$. Nos testes físicos foram avaliadas as mesmas 73 crianças nos cinco testes, obtendo um total de 365 resultados, identificando oito resultados "excelentes" (2,19\%), 52 "muito bons" (14,25\%), 60 "bons" (16,44\%), 73 "razoáveis" (20\%) e 172 "fracos" $(47,12 \%)$.

Dentre os oito resultados "excelentes", quatro foram no teste de força explosiva de membros superiores (arremesso de medicineball) e quatro no teste de aptidão cardiorrespiratória (corrida/caminhada de 6 minutos). 


\section{DISCUSSÃO}

A escola é o local onde todas as crianças frequentam e estão disponíveis para práticas corporais das mais diversas. Desta forma, Bandeira (2008) cita a escola como um ótimo local para a descoberta de jovens talentos. Entretanto, o autor lamenta a não existência de um plano de ação, seja governamental ou privado, que impeça que esses talentos sejam perdidos e que não dependa apenas da "boa vontade" do profissional de Educação Física encaminhá-los a clubes, escolinhas ou projetos para que sejam desenvolvidos.

Já Perfeito (2011) contribui afirmando que é um dos papéis do professor de Educação Física, ao perceber que algum aluno possui aptidões acima da média para alguma modalidade, convidá-lo ou encaminhá-lo para realizar um treinamento diferenciado, em horário extra classe, com exercícios voltados para o aprimoramento de suas técnicas e características físicas em algum local que realize atividades com esta finalidade. $\mathrm{O}$ autor afirma também que o objetivo principal da escola não é o descobrimento do jovem talento, mas o professor deve estar atento para esta possibilidade.

Mesmo considerando as médias dos resultados para cada idade como referência, é importante que o professor observe que uma criança pode apresentar uma variação de até três anos no desenvolvimento físico (BARBANTI, 2005). Existem casos de crianças que se destacam em uma faixa etária devido ao desenvolvimento diferenciado em relação aos seus colegas naquele período da vida, mas não conseguem se destacar posteriormente em idades mais avançadas, pois suas capacidades físicas acabam se igualando aos colegas que se desenvolveram mais tardiamente.

Para uma avaliação mais fidedigna, Ré (2011) indica que deveria ser considerada essa maturação nos indicativos da idade e sugere o teste de Tanner para complementar qualquer tentativa de detecção de talentos. Dantas, Portal e Santos (2004) afirmam que os talentos só podem ser detectados através de vários testes que se complementem e, após isso, criado para cada um deles um plano de expectativa individual. Além disso, Rega, Soares e Bojikian (2008) trazem as capacidades coordenativas como elemento fundamental para $\mathrm{o}$ desenvolvimento de qualquer outra habilidade motora e posicionam-se a favor da sua consideração para qualquer tentativa de detecção de talentos para o esporte.

No caso deste estudo, que separou os alunos pela faixa etária e sexo, conforme PROESP-BR (2016), alguns alunos foram classificados neste momento de suas vidas como possíveis talentos, sobretudo para capacidades como força explosiva de membros superiores e aptidão cardiorrespiratória. Além disso, a estatura se sobressaiu em três casos muito específicos quando comparados ao restante de seus colegas de escola.

Para os resultados expressivos obtidos nos testes de força explosiva de membros superiores, Bompa (2002) sugere um direcionamento desses alunos 
para modalidades que trabalhem essas características, como por exemplo, modalidades do atletismo como arremesso de peso e lançamentos de dardo e disco. Este autor lembra que esportes como arremessos e lançamentos no atletismo contam com atletas de estatura alta e fortes, com força de membros superiores. Já para os que se destacaram na aptidão cardiorrespiratória, indicam-se corridas com distâncias mais longas, que exigem maior resistência cardiorrespiratória e muscular dos membros inferiores. Nas corridas de fundo e marcha atlética se destacam a alta capacidade de resistência. Já o futebol exige coordenação, resistência à fadiga, alta capacidade aeróbia e anaeróbia.

A estatura também é um fator determinante para descobrir um talento para algumas modalidades, as quais esta característica é essencial. Bompa (2002) classifica os esportes coletivos como o basquetebol e o voleibol como tendo a necessidade de contar com atletas altos, com braços longos, alta capacidade aeróbia e anaeróbia, coordenação e resistência à fadiga.

Entretanto, nesta pesquisa o que chamou mais a atenção não foram os talentos, mas sim a quantidade de resultados classificados (de acordo com valores de referência do PROESP-BR) como "fracos", que totalizam 47,12 $\%$. Além disso, também os valores de IMC que resultaram em "sobrepeso" $(32,88 \%)$ e "obesos" $(9,59 \%)$, se somados, atingem quase a metade das crianças que participaram dos testes.

As causas do aumento da obesidade são muitas, podendo ser pela alimentação inadequada, da falta de espaços de lazer a partir do crescimento urbano ou o excesso de tempo no uso de aparelhos eletrônicos (DAMASO, 2001; MANCINI, 2002). As crianças de hoje optam por gastar menos energia, trocam as práticas de esportes e atividades físicas por outras atividades mais sedentárias, como uso da internet, jogos de vídeo game, programas de televisão e outros, se tornando cada vez mais inativas (MENDONÇA; ANJOS, 2004). Além disso, estudos apontam para uma tendência que crianças obesas continuem obesas quando adultas (FILADELFO, 2004).

\section{CONCLUSÃO}

O presente estudo detectou uma minoria de possíveis talentos em detrimento de uma maioria de alunos com resultados considerados "fracos" nos testes realizados, além dos altos números de sobrepeso e obesidade. Sugerese que, com o público avaliado, deva-se realizar um trabalho mais voltado para a saúde, participação e popularização da atividade física e/ou esportiva. Aos possíveis talentos detectados, sugere-se despender uma atenção ao seu direcionamento as modalidades mais indicadas para eles.

Sabe-se que o presente estudo apresenta limitações em quantidade de crianças avaliadas e sustentações teórico práticas para a definição do Talento Esportivo para além da idade somente. Ainda assim, parece ser válida a tentativa de aplicação de um protocolo existente no Brasil frente a inexistência 
de qualquer forma sistemática de deteç̧ão de talentos. Sabe-se que o PROESPBR propõem um protocolo de avaliações e valores de referência, mas isso não é o suficiente para considerar-se um sistema de identificação.

\section{REFERÊNCIAS}

BANDEIRA, T. L. Os futuros talentos esportivos estão nas aulas de educação física. Mas quem são eles? Rio de Janeiro: Universidade do Futebol, 2008.

BARBANTI, V. J. Formação de esportistas. São Paulo: Editora Manole, 2005.

BENDA, R. N. A deteç̧ão, seleção e promoção de talento esportivo em uma abordagem sistêmica. In: GARCIA, E. S. ET al. (Eds.). Temas atuais III: educação física e esportes. Belo Horizonte: Health, 1998, p. 52-78.

BÖHME, M. T. S. Aptidão Física de Jovens Atletas do Sexo Feminino Analisada em Relação a Determinados Aspectos Biológicos, Idade Cronológica e Tipo de Modalidade Esportiva Praticada. 1999. 123p. Tese (professor livre docente) Programa de Pós graduação em Educação Física - Escola de Educação Física, Universidade de São Paulo.

BOMPA, T. O. Periodização: teoria e metodologia do treinamento. São Paulo: Phorte Editora, 2002.

BRASIL. Projeto Esporte Brasil: PROESP-BR. Manual de testes e avaliação. Brasília: Ministério do Esporte. 2016.

DAMASO A. Nutrição e exercício na prevenção de doenças. $1^{a}$ ed. Rio de Janeiro: MEDSI, 2001.

DANTAS, E.; PORTAL, M. N. D.; SANTOS, L. A. V. Plano de expectativa individual:uma perspectiva científica para a detecção de talentos esportivos. R. Min. Educ. Fís., Viçosa, v. 12, n. 2, p. 72-100, 2004.

FILADELFO, L. Brasil: mais de 5 milhões de crianças obesas. $1^{\circ}$ Ed. São Paulo: Grande $\mathrm{ABC}, 2004$.

MANCINI, M. C. Métodos de avaliação de obesidade e alguns dados epidemiológicos. Rev. Abeso, Belo Horizonte, v.58, n.10, p, 127-132, 2002.

MENDONÇA, C. P.; ANJOS, L. A. Aspectos das Práticas Alimentares e da Atividade Física como Determinantes do Crescimento do Sobrepeso/Obesidade no Brasil. Cad. Saúde Publica, Rio de Janeiro, v.20, n.3, p. 32-45, 2004.

PERFEITO, R. S. O treinamento desportivo escolar e a revelação de jovens talentos. Lecturas, Educación Física y Deportes. Buenos Aires, nº 152, v.1, p.135-150, 2011. 
QUEIROZ, A. M.; FERNÁNDEZ, L. C.; Detecção de talentos na escola: possibilidades e limitações. Lecturas, Educación Física y Deportes, Buenos Aires, no 144, p 17-30, 2010 .

RÉ, A. H. N. Crescimento, maturação e desenvolvimento na infância e adolescência: Implicações para o esporte. Revista Motricidade, São Paulo, vol. 7, n. 3, p. 55-67, 2011.

REGA, G. C.; SOARES, T. A. A.; BOJIKIAN, J. C. M. Desenvolvimento das capacidades coordenativas no voleibol. Revista Mackenzie de Educação Física e Esporte, Belo Horizonte, v3, n7, p. 91-96, 2008.

ROTHER, R. L.; MEJIA, M. R. G. Treinamento de longo prazo e desenvolvimento humano: interfaces bioecológicas no esporte. Lecturas Educación Física y Deportes, Buenos Aires. n. 202, p. 102-115, 2015. 